\title{
La evaluación holística de la investigación en las artes en España: la necesidad y complejidad de confluir las dinámicas de los campos académico y artístico*
}

\author{
THE HOLISTIC ASSESSMENT OF RESEARCH IN THE ARTS IN SPAIN: \\ THE NEED AND COMPLEXITY OF CONVERGING THE DYNAMICS OF THE ARTISTIC \\ AND ACADEMIC FIELDS \\ A AVALIAÇÃO HOLÍSTICA DA PESQUISA NAS ARTES NA ESPANHA: \\ A NECESSIDADE E COMPLEXIDADE DE CONFLUIR AS DINÂMICAS DOS CAMPOS \\ ACADÊMICO E ARTÍSTICO
}

\section{Juan Arturo Rubio Arostegui** Raquel Caerols Mateo***}

Cuadernos de Música, Artes Visuales y Artes Escénicas

/ Volumen 13- Número 2 / Julio - Diciembre de 2018

/ ISSN 1794-6670/ Bogotá, D.C., Colombia / pp. 237-256

Fecha de recepción: 19 de octubre de 2016

Fecha de aceptación: 21 de junio de 2017

Disponible en línea: 1 de junio de 2018

doi: 10.11144/javeriana.mavae13-2.lehd

* Artículo de investigación. Este artículo es resultado de los proyectos Creación y estudios de las CAAC (colecciones y archivos de arte contemporáneo) de Cuenca como modelo metodológico para una investigación de excelencia en bellas artes, adscrito a la Facultad de Bellas Artes de la Universidad de Castilla-La Mancha, y Las colecciones de arte electrográfico y digital del MIDE: gestión, conservación, restauración y divulgación de sus fondos, adscrito a la Universidad de Bellas Artes de Cuenca. Asimismo, se ha realizado en el marco de los proyectos MINECO (Proyecto I+D+I Referencia: har2013-48604-c2-1-p) y PRINCET (Referencia: POII-2014-002-P) de la Facultad de Bellas Artes de la Universidad de Castilla-La Mancha, cuyo investigador principal es el catedrático José Ramón Alcalá Mellado.

* Licenciado en Filosofía y Ciencias de la Educación por la Universidad Complutense de Madrid, magíster en Gestión Cultural y doctor en Ciencias Políticas y Sociología por la Universidad Complutense de Madrid. Director de la Escuela de Doctorado en la Universidad Nebrija. ORCID: 0000-0002-7236-2866

*** Licenciada en Comunicación Audiovisual por la Universidad Complutense de Madrid y doctora en Creatividad aplicada por la Universidad Complutense de Madrid. Profesora adjunta de Creatividad y Artes Visuales en los grados de Comunicación y Bellas Artes en la Universidad Francisco de Vitoria. ORCID: 0000-0001-5167-8629 


\section{Resumen}

La naturaleza epistemológica y del arte y sus modos de hacer determinan las formas de evaluar la calidad de la investigación en esta disciplina. La coexistencia de diferentes modos de definir la investigación en las artes y sus enfoques complejiza las formas institucionales de evaluar la investigación, pues se constatan nuevas formas de investigar como consecuencia del giro performativo en las ciencias sociales. En esta situación, los profesores de artes en las universidades españolas encuentran dificultades para articular sus prácticas creativas y solventar sus resultados de investigación con las agencias de evaluación del sistema universitario, lo cual tiene como consecuencia una debilidad en su capital académico-investigador y una evidencia en los indicadores de la cienciometría. La teoría de los campos de Bourdieu nos permite tanto analizar las dinámicas de los campos académico y artístico como plantear posibles soluciones teóricas y prácticas para una mejor evaluación institucional de la investigación. Para abordar la problemática descrita, nos planteamos un análisis cualitativo de la dimensión epistemológica y académica de la cuestión, y otro cualitativo centrado en los indicadores de calidad de las principales bases de datos. Detectadas las deficiencias y las incongruencias del modelo académico y el perfil del profesorado de las artes, apuntamos la necesidad de plantear un modelo desarrollado por los decanos de las facultades de bellas artes pero que no ha sido aplicado a dichas facultades.

Palabras clave: evaluación de la investigación; agencialización; investigación en las artes; artes visuales.

\section{Abstract}

The epistemological nature and that of the art and its ways of acting, determine the forms of assessing the quality of research in this discipline. The co-existence of different modes of defining research in arts and its approaches make the institutional forms of assessing research more complex, because they validate new forms of conducting research as a consequence of the performative shift in social sciences. Given this situation, art professors at Spanish universities find difficulties to articulate their creative practices and to solve their research results with the assessment agencies of the university system, which results in a weakness in terms of their scholar-research capital and an evidence of the indicators of the scientometrics. Bourdieu's field theory allows us to analyze the dynamics of the academic and artistic fields, as well as to propose possible theoretical and practical solutions for a better institutional eva- luation of research. To address the problem described, we set out a qualitative analysis of the epistemological and academic dimension of the issue, and a qualitative analysis focused on the quality indicators of the main databases. Once the deficiencies and inconsistencies of the academic model and the profile of the teachers of the arts were detected, we pointed out the need to propose a model developed by the deans of faculties of fine arts but that has not been applied to these faculties.

Keywords: evaluation of the investigation; agentization; research in the arts; visual arts.

\section{Resumo}

A natureza epistemológica e da arte e seus modos de realização determinam as formas de avaliar a qualidade da pesquisa nesta disciplina. A coexistência de diferentes modos de definir a pesquisa nas artes e suas abordagens tornam mais complexas as formas institucionais de avaliar a pesquisa, pois constatam-se novas formas de pesquisar como consequência do giro performativo nas ciências sociais. Nesta situação, os professores de artes nas universidades espanholas encontram dificuldades para articular suas práticas criativas e solucionar seus resultados de pesquisa com as agências de avaliação do sistema universitário, o qual tem como consequência uma debilidade em seu capital académico-pesquisador e uma evidência nos indicadores da cienciometria. A teoria dos campos de Bourdieu nos permite, tanto analisar as dinâmicas dos campos académico e artístico, quanto abordar possíveis soluções teóricas e práticas para uma melhor avaliação institucional da pesquisa. Para abordar a problemática descrita, nos planteamos uma análise qualitativa da dimensão epistemológica e acadêmica da questão, e outra qualitativa focada nos indicadores de qualidade das principais bases de dados. Detectadas as deficiências e as incongruências do modelo acadêmico e o perfil do professorado das artes, destacamos a necessidade de sugerir um modelo desenvolvido pelos decanos das faculdades de belas artes mas que não tenha sido aplicado a essas faculdades.

Palavras-chave: avaliação da pesquisa; agencialização;

pesquisa nas artes; artes visuais. 


\section{INTRODUCCIÓN: LA NATURALEZA EPISTEMOLÓGICA DEL ARTE}

Una de las cuestiones sustantivas sobre la que descansa una buena parte de la situación de las artes en el campo académico es aquella que se refiere a la naturaleza del conocimiento que genera la experiencia del arte, por un lado, y la que se adhiere a la naturaleza del saber y del saber hacer del artista, por otro. El arte es una de las formas que tiene el hombre del conocer el mundo (Eisner 2008), en el que el componente emocional tiene papel protagónico. El arte muestra aspectos del mundo; no explica como lo hace la ciencia (Dewey 2008) ofreciendo un conocimiento del mundo de modo cualitativo y empático. Tanto Dewey como Eisner defienden la tesis de que la experiencia del arte es una particular forma de conocimiento del hombre. Asimismo, la noción de experiencia estética, además de remitir a la recepción artística, "está conectada de modo inherente a la experiencia de hacer" de un artista (Dewey 2008, 15).

Pero no ha sido hasta el siglo XX cuando en las humanidades y las ciencias sociales se plantea la experiencia del arte como conocimiento $y$, a su vez, los procesos de saber y del saber hacer con el ethos de la ciencia. El arte, en cuanto praxis, fue asimilado desde Grecia a la téchnē y, por tanto, a un saber no ligado a la epistếmē. Por un lado, la téchnē es definida como un conjunto de conocimientos prácticos para poder crear. Por otro, el saber del arte no ligado a la epistếmē supone el inicio de una dependencia histórica en cuanto se escapa de los condicionamientos filosóficos y científicos de la epistếmē (conocimiento). De hecho, la teoría del conocimiento en la filosofía — desde Platón y Aristóteles, pasando por la disputa entre racionalistas y empiristas en la modernidad sobre el origen de las ideas, hasta Habermas - no tematiza la especificidad y naturaleza del saber artístico y su experiencialidad.

Como decíamos, es el siglo XX, en el marco de los procesos de creación de científicos y artistas, cuando se incluyen algunos aspectos del método científico al evidenciarse las primeras analogías sobre el ethos de los artistas y los científicos en cuanto a sus actitudes y sus métodos formulados por Dewey:

La noción extravagante de que un artista no piensa y que un investigador científico no hace otra cosa que pensar, resulta de convertir una diferencia de tempo y énfasis en una diferencia de calidad. El pensador tiene su momento estético, cuando sus ideas dejan de ser meras ideas y se convierten en el significado corpóreo de los objetos. El artista tiene sus problemas y piensa al trabajar, pero su pensamiento está más inmediatamente incorporado al objeto. Debido a que su objetivo está más alejado, el científico opera con símbolos, palabras y signos matemáticos. El artista realiza su pensamiento en los medios cualitativos mismos con que trabaja, y sus fines se encuentran tan cerca del objeto que produce que se funden directamente de él. $(2008,17)$

En el siglo XXI, evidenciamos estas analogías en análisis de casos como el que propone Miller (2007) en los casos de Einstein y Picasso. Miller realiza un análisis comparativo entre los procesos de creación en la bifurcación formada en lo que se entienden como dos ámbitos del saber, arte o ciencia, de todas las fases que están tipificadas e identificadas en los procesos del pensamiento creativo: búsqueda, punto muerto, insight y resultado, que tiene su significación y traducción en la verificación. Es ya sabido que los resultados en la ciencia solo son válidos si existe un proceso de verificación. 
Miller fundamenta la verificación en el arte, definiendo las claves que cimentan esta verificación. Identifica tres clases de verificación, de las cuales, dos son específicas de la ciencia: "la teoría no debe ir en contra de los datos empíricos" (2007, 301). La segunda tiene que ver con el proceso que siguió Einstein en su proceso de producción de novedad, "optar por teorías 'cuyo objetivo es la totalidad de las apariencias físicas'" (301). El tercero lo precisa así:

El tercer método de evaluación, que se aplica tanto al arte como a la ciencia, es aún más sutil. Es la verificación mediante la influencia: ¿ha llevado la idea a alguna parte?, ¿inspira a otros a producir una ciencia útil o un arte de importancia?, ¿se convierte en parte de una visión del mundo? Está claro que la teoría de la relatividad de Einstein y Las señoritas de Aviñón de Picasso cumplen todos esos requisitos. [...] nunca podrán olvidarse, porque ya forman parte de la piedra sobre la que siempre se asentarán la ciencia y el arte en su conjunto. (302)

\section{EL GIRO PERFORMATIVO EN LAS CIENCIAS SOCIALES Y SUS CONSECUENCIAS EN LA INVESTIGACIÓN EN LAS ARTES}

La sociología de la educación superior y la sociología del conocimiento han tenido como temas de estudio la coincidencia en una misma época de definiciones distintas de lo que es investigar y de sus modos, a través de las diferentes metodologías (Bourdieu 2008; Collins 2000). Esta coexistencia de definiciones y modos de la praxis investigadora — que es el hecho de investigar- es más evidente en el caso de las humanidades y las ciencias sociales que en el resto de las ciencias. Pero es en el campo de las artes donde más claramente se produce una coexistencia de enfoques de investigación más tradicionales producidos en su mayor parte por la historia del arte con otros emergentes, surgidos de la práctica artística como investigación.

El giro peformativo (performative turn) en las ciencias sociales, no solo ha traído unas nuevas formas de definir y hacer investigación en las ciencias sociales y las humanidades, sino que se ha instalado como una disciplina (performance studies), tal como reconoce y categoriza el editor académico Routledege. En el caso de las artes, el giro performativo ha permitido que la práctica artística, en sus procesos y en su praxis, forme parte del objeto de investigación. Hay numerosos autores que hablan de un conocimiento que puede ser mostrado y articulado a través de la experimentación e interpretación, que se genera en la práctica artística como consecuencia de haber llevado a cabo una investigación original (Borgdorff 2010).

En la actualidad, podemos establecer la coincidencia en el tiempo de distintos modos de investigar en las artes como consecuencia del proceso de sedimentación que se da entre los modos tradicionales de las disciplinas con más recorrido histórico, como la historia del arte o la estética, y el aluvión de nuevos enfoques surgidos de la amalgama que se da dentro del paradigma constructivista de las ciencias sociales. De ello, se hacen cargo los "Indicadores para la evaluación de la actividad investigadora en los ámbitos de las bellas artes" (2011) que plantea las siguientes dimensiones: 1) la investigación basada en el proyecto, 2) la investigación basada en la práctica artística y 3) la investigación basada en el pensamiento. Esta tercera dimensión tiene que ver con las formas de investigación tradicionales en el ámbito académico. La investigación basada en el proyecto y la investigación 
basada en la práctica artística se corresponderían con la téchnē y la investigación basada en el pensamiento estaría asimilada al conocimiento científico tradicional. La importancia del giro performativo en el paradigma constructivista va más allá del concepto de razón histórica formulado por Ortega y Gasset: "Frente a la razón pura físico-matemática hay, pues, una razón narrativa. Para comprender algo humano, personal o colectivo, es preciso contar una historia" (2006, 40). El paradigma constructivista introduce la subjetividad y el uso del lenguaje como un elemento conformador de la realidad, y la narratividad forma parte de la interacción entre el investigador y el sujeto investigado, pues es lo que permite el estudio de fenómenos sociales, las experiencias, la significación y las representaciones simbólicas del mundo que se dan desde distintos individuos y subsistemas sociales. Hoy en día, en las ciencias sociales, conviven dos paradigmas (positivista y constructivista) a la hora de enfocar la investigación social, y es en el constructivista donde se instala la mayor parte del conocimiento de las artes. Y es, precisamente, en la naturaleza de la téchnē del arte, en esa praxis, donde se han ubicado las nuevas formas de investigación en las artes (en el proyecto y en la praxis artística) bajo el paraguas del giro performativo acaecido a finales del siglo XX.

Las primeras propuestas que tratan de contextualizar la práctica artística al vincularla con la epistemología en España provienen de Hernández (2008), las cuales recogen la tradición anglosajona de autores como Caduff, Siegenthaler y Wälchli (2010) o Sullivan (2004) quienes vinculan la práctica artística del taller como formas legítimas de investigar y otros que relacionan la investigación en el arte con la terapia en el campo educativo utilizando

procedimientos artísticos (literarios, visuales y performativos) para dar cuenta de prácticas de experiencia en las que tanto los diferentes sujetos (investigador, lector, colaborador) como las interpretaciones sobre sus experiencias desvelan aspectos que no se hacen visibles en otro tipo de investigación. (Hernández 2008, 92-93)

Estos enfoques surgidos de la literatura académica anglosajona se denominan artistic research (investigación artística) y arts-based research (investigación basada en las artes). Ahora bien, hay que destacar en todo este proceso histórico que hemos tratado de esbozar sucintamente la aportación de Eisner a lo largo de los siglos XX y XXI, pues es central a la hora de vincular arte y conocimiento, junto con los primeros textos iluminadores de Dewey desde la década de 1930.

\section{LA VINCULACIÓN DE LAS ENSEÑANZAS DE ARTE AL CAMPO ACADÉMICO: CONTEXTO Y CONSECUENCIAS EN EL CASO ESPAÑOL}

En 1978, las escuelas de bellas artes en España pasan a ser facultades, lo cual se traduce en el hecho de que estas enseñanzas pasan al espacio universitario con una ley orgánica distinta de la que les regulaba con anterioridad. ${ }^{1}$ El cambio al campo académico conlleva un cambio del statu quo del artista docente, pues pasan a ser sometidos, al igual que en otros campos de conocimiento, a la evaluación de su actividad investigadora como docentes de la educación superior. Es curioso como Bourdieu (2008) ejemplifica y define la condición social del profesor universitario por oposición a la de artista en su obra Homo academicus: 
Los profesores universitarios se sitúan más bien del lado del polo dominado del campo del poder y se oponen claramente a ese respecto a los patrones de la industria y del comercio. Pero, en tanto que poseedores de una forma institucionalizada de capital cultural, que les asegura una carrera burocrática e ingresos regulares, se oponen a los escritores y a los artistas: ocupando una posición temporalmente dominante en el campo de producción cultural, se distinguen por ello, en grados diversos según las facultades, de los ocupantes de los sectores menos institucionalizados y más heréticos de ese campo (y especialmente de los escritores y de los artistas, a los que se llama "libres" o "free lance" por oposición a aquellos que pertenecen a la universidad. (54)

La cuestión clave de los profesores artistas adscritos a las facultades de bellas artes es la debilidad de los distintos tipos de capitales culturales académicos, propios de los habitus de los profesores de otras facultades más orientados a las lógicas y las dinámicas de la investigación (caso de las facultades de ciencias y algunas de humanidades y ciencias sociales) o la tendencia de posiciones de poder en el caso de la medicina y el derecho o, asimismo, la pertenencia a escuelas superiores de élite como las que se desarrollaron a mediados del siglo XX en Francia (no obstante, en España, no podemos evidenciar, tal como ocurre en el caso francés, este conglomerado de instituciones superiores de educación que ordenan el reconocimiento de los estudiantes y profesores). Los profesores artistas han podido acceder a las instituciones educativas como consecuencia de sus méritos artísticos y académicos, pero, tal como veremos más adelante con las evidencias de la bibliometría y la cienciometría, la debilidad de su capital académico-investigador les impide obtener un reconocimiento (en forma de sexenios o la posibilidad de ser catedráticos, por ejemplo) por parte de las agencias evaluadoras del sistema universitario español (instancias públicas e institucionalizadas de reputación y reconocimiento y sanción para las universidades, los títulos y los profesores). El capital académico-investigador define cada posición en el campo académico y se manifiesta en su posición con respecto a las publicaciones en revistas académicas indexadas y en la indexación de las editoriales de libros, las citas en su disciplina académica, el reconocimiento internacional de su investigación a través de traducciones, la pertenencia a comités de revistas académicas de prestigio o la consagración a través de la notoriedad y vinculación en los medios de comunicación social.

Pero, si bien este statu quo apenas varió desde la incorporación de las facultades de bellas artes a las universidades públicas y la posterior oferta a partir de mediados de la década de 1990 de las universidades privadas, no es hasta inicios del siglo XXI cuando la cuestión de la evaluación de la investigación en el campo de las artes y, en concreto, en el de las bellas artes se plantea como algo problemático que afecta el conjunto del profesorado. El motivo de este cambio en el siglo XXI es debido también al proceso de agencialización del campo académico. La agencialización consiste, según Talbot et al. (2000) en la constitución de agencias públicas especializadas, con el objetivo de separar el rol de principal y agente, es decir, en separar la toma de decisiones y la capacidad de gestionar, especificando claramente los objetivos y los medios para conseguirlos. De esta forma, las unidades gestoras pueden devenir más eficientes, transparentes y responsables de sus acciones (Serra 2007). Se trata de un fenómeno global en el campo académico. En nuestro país, este proceso se ha desplegado en un tiempo relativamente corto - a partir de la última década del siglo XX — a través de un conjunto de entidades públicas que fomentan una internacionalización y la aplicación de indicadores que miden la calidad de la ciencia española. De estas entidades públicas, podemos destacar la Agencia Nacional de Evaluación 
y Prospectiva (ANEP) que tiene como principal objetivo evaluar la calidad científico-técnica de los proyectos de investigación que solicitan financiación pública en concurrencia competitiva (planes nacionales de I+D+I); la Fundación Española para la Ciencia y la Tecnología cuya misión es impulsar la ciencia e innovación promoviendo su integración y acercamiento a la sociedad, apoyando en el ámbito de sus funciones las necesidades de los agentes del Sistema Español de Ciencia, Tecnología e Innovación; la Comisión Nacional Evaluadora de la Actividad Investigadora que realiza la evaluación de la actividad investigadora de los profesores universitarios y del personal de las escalas científicas del Consejo Superior de Investigaciones Científicas (CSIC), con el objeto de que les sea reconocido un complemento de productividad (sexenios); la Agencia Nacional de Evaluación de la Calidad y Acreditación (Aneca) que tiene como objetivo contribuir a la mejora de la calidad del sistema de educación superior mediante la evaluación, certificación y acreditación de enseñanzas (grado, máster y doctorado), profesorado e instituciones; y las agencias de calidad autonómicas que también velan por la acreditación y certificación en su ámbito territorial y que dan una idea del entramado institucional del proceso de agencialización en el campo académico en el Estado español. Este contexto agencializado ha potenciado aceleradamente las dinámicas que ya existían, entre ellas la distinción y competitividad, en el cual la evaluación de la investigación polariza los reconocimientos (campus de excelencia, doctorados con mención de calidad, entre otros) y prevalece con respecto a otros posibles criterios en la evaluación de la idoneidad del profesorado, la verificación y acreditación de los títulos universitarios y la asignación de fondos para los grupos de investigación a través de las convocatorias en concurrencia competitiva (Sanz 2014). Además de la agencialización, la aparición de los rankings de universidades a nivel internacional tiende a incrementar aún más las diferencias de reputación académica entre instituciones y países. Sus indicadores cuantitativos posicionan y clasifican el campo académico y cada vez son más tenidos en cuenta por los gobiernos, las propias agencias y las universidades. Todo este sistema de valoración que se basa en las reglas del campo académico no está exento de críticas desde los propios académicos. Así, Sacristán (2014) identifica algunos efectos perversos del factor de impacto que se observa en la conducta investigadora del profesorado que afecta también al progreso de la ciencia:

La proliferación de publicaciones, el troceamiento de los resultados de la investigación y la escasez de trabajos de alcance amplio, realmente iluminadores. En pocas palabras, el sistema está tendiendo a la cantidad, más que a la calidad y a la relevancia de la investigación. Este es un fenómeno contrario al interés general del avance científico. (152)

No obstante, es difícil pensar que a corto plazo estas dinámicas que evalúan el rendimiento a través de indicadores cuantitativos puedan cambiar, ya que esto afecta también la forma de medir la calidad de la educación obligatoria por países a través de indicadores como el de PISA (Programme for International Student Assessment). Sin embargo, podemos constatar que en España han surgido intentos de medir la calidad investigadora en las humanidades y las ciencias sociales con nuevas metodologías en la Universidad del País Vasco (Barrenechea, Castro e Ibarra 2009). El propósito de estos intentos es el de medir la actividad investigadora con indicadores cuantitativos y cualitativos y de un modo más holístico y relacional, que sean adecuados con el ethos de estos campos de conocimiento. 


\section{LA INVESTIGACIÓN COMO PENSAMIENTO Y CONOCIMIENTO: LA EVALUACIÓN DE LA INVESTIGACIÓN EN LAS ARTES A TRAVÉS DE LAS REVISTAS ACADÉMICAS}

En España, la evaluación de la investigación, tomando como referencia las publicaciones científicas en las revistas académicas, comienza en la década de 1990 cuando se tiene constancia de un primer estudio desde el ámbito de la cienciometría y la documentación en los campos de la sociología, arqueología, prehistoria e historia antigua (Alcaín, Román y Giménez 2008). Por otro lado, la actividad investigadora en el campo de las bellas artes se materializa a través de distintos canales de comunicación, entre los cuales la publicación en revistas de carácter científico y académico no alcanza la extensión y relevancia que se observa en otras áreas de las humanidades y las ciencias sociales (Pastor et al. 2013). Este trabajo referenciado concluye que, en el caso de la investigación en las bellas artes, las publicaciones periódicas académicas de la disciplina no cumplen con la función de ser un canal de comunicación entre la comunidad académica. Así, del conjunto de profesores de la universidad que analiza la muestra, son muy pocos los que publican y con muy baja frecuencia (la media es de 1.9 artículos por profesor en el periodo 1977-2010). Asimismo, los profesores publican en otras publicaciones periódicas englobadas en el conjunto de revistas culturales que no cumplen - o cumplen muy poco- con los criterios -el más importante, la revisión anónima de los pares- de las revistas académicas, si bien desde 2002 se constata un aumento de las publicaciones como consecuencia — según los autores- de "los criterios marcados por la Aneca [Agencia Española de Evaluación del Sistema Universitario] y la CNEAI [Comisión Nacional Evaluadora de la Actividad Investigadora]." Esta evidencia cuantitativa es la que principalmente define el capital académico-científico del profesorado adscrito a las facultades de bellas artes, tal como observábamos en párrafos anteriores, y eso tiene un efecto directo y negativo en su reputación y reconocimiento por parte de las agencias del sistema universitario español. Esta situación es la que provoca que el conjunto de los decanos de las facultades de bellas artes considere la necesidad de analizar este escenario en los "Indicadores para la evaluación de la actividad investigadora en los ámbitos de las bellas artes" (2011).

\section{METODOLOGÍA}

Planteada esta cuestión, y tras el análisis cualitativo sobre ellas, nos proponemos llevar a cabo un análisis cuantitativo centrado en el análisis de los indicadores de calidad en el ámbito de las bases de datos y la bibliometría, que nos lleve a ser conscientes y tener evidencias de las problemáticas actuales planteadas en dicho análisis.

Este análisis cuantitativo está centrado, pues, en la tercera dimensión que establecen los decanos de las facultades de bellas artes en los "Indicadores para la evaluación de la actividad investigadora en los ámbitos de las bellas artes" (2011), que corresponde con los sistemas tradicionales de investigación del ámbito académico y, por tanto, a estas bases de datos y la bibliometría. 
Tomamos como referencia las bases de datos más relevantes nacionales e internacionales:

- DICE (Difusión y Calidad Editorial de las Revistas Españolas de Humanidades y Ciencias Sociales y Jurídicas)

- RESH (Revistas Españolas de Ciencias Sociales y Humanidades)

- IN-RECH (Índice de Impacto Revistas Españolas de Ciencias Humanas)

- CIRC (Clasificación Integrada de Revistas Científicas)

- Scopus

Respecto de estas bases de datos, atenderemos a los siguientes parámetros:

- Área temática

- Número de revistas

- Año de comienzo de revista más antigua

- Porcentaje de cumplimiento de periodicidad

- Porcentaje de evaluadores externos

- Porcentaje de revistas incluidas en Latindex

- Porcentaje de revistas incluida en alguna base de datos internacional

Este análisis de datos se hará por periodos o tramos de cuatro años (1999-2003, 20042008, 2009-2013), así como 2015 (siempre que los datos estén actualizados), a partir de los cuales se pretende hacer un mapa de cómo han evolucionado las revistas de investigación en las artes: revistas aparecidas y desaparecidas, media de permanencia de las revistas, posicionamiento e índices de impacto, etc.

Para el análisis cuantitativo, seguiremos el patrón establecido en el epígrafe de metodología. De tal manera que analizaremos el ámbito de las artes, las visual arts y performing arts, según las diferentes denominaciones de las distintas bases de datos seleccionadas para el presente estudio.

Establecido el ámbito de conocimiento por analizar, contemplamos el análisis de las principales bases de datos como indicadores de calidad de tipología bibliométrica (revistas) de las artes.

\section{RECOGIDA Y ANÁLISIS DE DATOS}

\section{DICE (DIFUSIÓN Y CALIDAD EDITORIAL DE LAS REVISTAS ESPAÑOLAS DE HUMANIDADES Y CIENCIAS SOCIALES Y JURÍDICAS)}

Fecha de creación: 2006

Grupo de Investigación Evaluación de Publicaciones Científicas en Ciencias Sociales y Humanas del Instituto de Estudios Documentales sobre Ciencia y Tecnología del Centro de Ciencias Humanas y Sociales del CSIC (véase tabla 1). 


\begin{tabular}{|c|c|c|c|c|c|c|c|}
\hline & $\begin{array}{c}\text { Área } \\
\text { temática }\end{array}$ & $\begin{array}{l}\mathrm{N}^{\circ} \text { de } \\
\text { revistas }\end{array}$ & $\begin{array}{c}\text { Año comienzo } \\
\text { revista más } \\
\text { antigua }\end{array}$ & $\begin{array}{c}\% \\
\text { cumplimiento } \\
\text { periodicidad }\end{array}$ & $\begin{array}{c}\% \\
\text { evaluadores } \\
\text { externos }\end{array}$ & $\begin{array}{l}\% \text { de revistas } \\
\text { incluidas en } \\
\text { LATINDEX } \\
\text { (A partir de } 30 \\
\text { criterios) }\end{array}$ & $\begin{array}{l}\% \text { de revistas } \\
\text { incluidas en } \\
\text { alguna BB.DD } \\
\text { internacional }\end{array}$ \\
\hline 1 & Historia del arte & 63 & 1915 & & $\begin{array}{c}23 \text { de } 63 \\
36,5 \%\end{array}$ & $\begin{array}{c}18 \text { de } 63 \\
28,5 \%\end{array}$ & $\begin{array}{c}26 \text { de } 63 \\
41,3 \%\end{array}$ \\
\hline 2 & $\begin{array}{l}\text { Didáctica de la } \\
\text { expresión plástica }\end{array}$ & 3 & 1988 & & $100 \%$ & $100 \%$ & $100 \%$ \\
\hline 3 & $\begin{array}{l}\text { Didáctica de la } \\
\text { expresión música }\end{array}$ & 3 & 1995 & & $66,67 \%$ & $66,60 \%$ & $33,30 \%$ \\
\hline 4 & Música & 25 & 1946 & & $\begin{array}{c}9 \text { de } 25= \\
36 \%\end{array}$ & $\begin{array}{c}6 \text { de } 25= \\
24 \%\end{array}$ & $\begin{array}{c}8 \text { de } 25= \\
32 \%\end{array}$ \\
\hline 5 & $\begin{array}{l}\text { Biblioteconomía } \\
\text { y documentación }\end{array}$ & 3 & 1994 & & $33,30 \%$ & $33,30 \%$ & $33,30 \%$ \\
\hline 6 & Arqueología & 2 & 1986 & & $0 \%$ & $0 \%$ & $0 \%$ \\
\hline 7 & Dibujo & 1 & 2011 & & $100 \%$ & $100 \%$ & $100 \%$ \\
\hline 8 & $\begin{array}{c}\text { Estética y teoría } \\
\text { de las Artes }\end{array}$ & 3 & 2004 & & $33,30 \%$ & $66,60 \%$ & $0 \%$ \\
\hline 9 & $\begin{array}{c}\text { Comunicación Audiovisual } \\
\text { y publicación }\end{array}$ & 4 & 1989 & & $75 \%$ & $75 \%$ & $50 \%$ \\
\hline 10 & Antropología social & 1 & 1995 & & $100 \%$ & $100 \%$ & $100 \%$ \\
\hline 11 & $\begin{array}{l}\text { Composición } \\
\text { arquitectónica }\end{array}$ & 1 & 1998 & & $0 \%$ & $0 \%$ & $100 \%$ \\
\hline
\end{tabular}

Tabla 1. Datos globales de las revistas incluidas en el área temática de bellas artes (137 revistas) en DICE. (Único periodo de actualización: 2006-2010. Última actualización: 29-7-2010).

Fuente: Elaboración propia.

En primer lugar, queremos aclarar que, aunque están catalogadas en el ítem bellas artes 137 revistas, la recogida de datos por áreas temáticas solo suma 109, lo cual quiere decir que 28 no están incluidas en ninguna de las categorías establecidas. Dicho esto, tras esta recogida de datos, el primer hecho que podemos constatar es la falta de actualización de esta base de datos estando aún vigente (2006-2010). La clasificación del campo de las artes se compone de una gran miscelánea de materias, en la cual quedan muy escasas la representación de las materias específicas de las artes, a saber: historia del arte, música, dibujo y estética y teoría de las artes. De estas cuatro materias, la única concreta que se acerca a la praxis de las artes es la de dibujo, que queda solo representada por una revista, cuya antigüedad es tan solo de 2011, con evaluadores externos, pero sin ninguna presencia en bases de datos internacionales. Sí podemos afirmar que la materia más representativa de todas es la de historia del arte; no obstante, este tipo de publicaciones sigue unas metodologías de investigación especialmente ortodoxas en relación con las tradicionales metodologías académicas. Asimismo, el porcentaje de evaluadores externos de estas es escaso, pues solo cuentan con ellos 21 revistas de 63, al igual que su presencia en bases de datos internacionales, que no llega ni a la mitad. 


\section{RESH (REVISTAS ESPAÑOLAS DE CIENCIAS SOCIALES Y HUMANIDADES)}

Inicia su actividad en 1999. En ese año, España se incorpora al Sistema Regional de Información para América Latina, el Caribe, España y Portugal (Latindex). Este sistema definió 33 criterios de "calidad editorial" para las revistas. Por ello, en el Centro de Información y Documentación Científica, se abordó el estudio de 1300 revistas españolas de humanidades y ciencias sociales, tras lo cual se llevó a cabo una categorización de estas según el cumplimiento de criterios de calidad editorial.

- Clasificación de las humanidades: arqueología y prehistoria, bellas artes, estudios árabes, hebreos y orientales, filología clásica, filologías hispánicas, filologías modernas, filosofía, historia antigua y medieval, historia del arte, historia general y especializada, historia moderna y contemporánea, lingüística general y aplicada.

- El índice de impacto medio (IIM), calculado a partir de los índices de impacto de 1999 a 2003 : analizaríamos solo el área en las que se incluyen las bases de datos, que consta de 122 revistas catalogadas (http://epuc.cchs.csic.es/resh/indicadores) (véanse tablas 2 y 3 ).

\begin{tabular}{|c|c|c|c|c|c|c|c|c|}
\hline $\begin{array}{c}\text { Área } \\
\text { temática }\end{array}$ & $\begin{array}{l}N^{\circ} \text { rev. } \\
\text { en } \\
\text { RESH }\end{array}$ & $\begin{array}{c}\text { Año comienzo } \\
\text { rev. más } \\
\text { antigua }\end{array}$ & $\begin{array}{c}\% \text { rev. } \\
\text { cumplimiento } \\
\text { periodicidad }\end{array}$ & $\begin{array}{l}\% \text { rev. con } \\
\text { evaluadores } \\
\text { externos }\end{array}$ & $\begin{array}{l}\text { Media } \\
\text { ind. } \\
\text { val. A+B }\end{array}$ & $\begin{array}{c}\% \text { de rev. } \\
\text { incluidas en } \\
\text { cat. LATINDEX }\end{array}$ & $\begin{array}{l}\% \text { de revistas } \\
\text { incluidas en } \\
\text { alguna BB.DD } \\
\text { internacional }\end{array}$ & $\begin{array}{c}\text { Material } \\
\text { índice impacto } \\
\text { medio }\end{array}$ \\
\hline América Latina & 19 & 1940- & 78,95 & 47,37 & 41,06 & 63,16 & 94,74 & 0,037 \\
\hline Antropología & 20 & $1944-$ & 65,00 & 35,00 & 26,68 & 50,00 & 68,42 & 0,024 \\
\hline Arqueología & 57 & $1928-$ & 38,60 & 14,04 & 40,70 & 45,61 & 47,37 & 00,67 \\
\hline Bellas Artes & 45 & 1915- & 51,11 & 20,00 & 34,12 & 31,11 & 60 & 0,027 \\
\hline Filosofía & 42 & $1901-$ & 76,19 & 33,33 & 31,18 & 52,38 & 76,19 & 0,027 \\
\hline Geografía & 29 & $1876-$ & 68,97 & 68,97 & 43,52 & 82,76 & 75,86 & 0,060 \\
\hline Historias & 127 & $1877-$ & 64,57 & 29,92 & 40,15 & 44,88 & 59,06 & 0,076 \\
\hline Ling. y Literatura & 92 & $1901-$ & 52,17 & 36,96 & 33,60 & 57,61 & 75,00 & 0,024 \\
\hline Misceláneas & 96 & $1922-$ & 50,00 & 12,50 & 40,32 & 29,17 & 38,54 & 0,025 \\
\hline
\end{tabular}

Tabla 2. Datos globales derivados de la valoración integrada y ponderación para las áreas de ciencias humanas (1999-2003). Fuente: Alcaín, Román y Giménez (2008). 


\begin{tabular}{|c|c|c|c|c|c|c|c|}
\hline & $\begin{array}{c}\text { Área } \\
\text { temática }\end{array}$ & $\begin{array}{l}\mathrm{N}^{\circ} \text { de } \\
\text { revistas }\end{array}$ & $\begin{array}{c}\text { Año comienzo } \\
\text { revista más } \\
\text { antigua }\end{array}$ & $\begin{array}{c}\% \\
\text { cumplimiento } \\
\text { periodicidad }\end{array}$ & $\begin{array}{c}\% \\
\text { evaluadores } \\
\text { externos }\end{array}$ & $\begin{array}{l}\% \text { de revistas } \\
\text { incluidas en } \\
\text { LATINDEX } \\
\text { (A partir de } 30 \\
\text { criterios) }\end{array}$ & $\begin{array}{l}\% \text { de revistas } \\
\text { incluidas en } \\
\text { alguna BB.DD } \\
\text { internacional }\end{array}$ \\
\hline 1 & Historia del arte & 53 & 1915 & & $\begin{array}{c}17 \text { de } 53= \\
32 \%\end{array}$ & $\begin{array}{c}19 \text { de } 53= \\
25,8 \%\end{array}$ & $\begin{array}{c}25 \text { de } 53= \\
47,1 \%\end{array}$ \\
\hline 2 & $\begin{array}{l}\text { Didáctica de la } \\
\text { expresión plástica }\end{array}$ & 2 & 1988 & & $\begin{array}{c}2 \text { de } 2= \\
100 \%\end{array}$ & $\begin{array}{c}2 \text { de } 2= \\
100 \%\end{array}$ & $\begin{array}{c}2 \text { de } 2= \\
100 \%\end{array}$ \\
\hline 3 & $\begin{array}{l}\text { Didáctica de la } \\
\text { expresión música }\end{array}$ & 3 & 1995 & & $\begin{array}{c}1= \\
33,3 \%\end{array}$ & $\begin{array}{c}2= \\
66,6 \%\end{array}$ & $\begin{array}{c}1= \\
33,3 \%\end{array}$ \\
\hline 4 & Música & 19 & 1946 & & $\begin{array}{c}10 \text { de } 19= \\
52,6 \%\end{array}$ & $\begin{array}{c}8 \text { de } 19= \\
42,1 \%\end{array}$ & $\begin{array}{c}9 \text { de } 19= \\
47,3 \%\end{array}$ \\
\hline 5 & $\begin{array}{l}\text { Biblioteconomía } \\
\text { y documentación }\end{array}$ & 3 & 1994 & & $\begin{array}{l}1 \text { de } 3= \\
33,3 \%\end{array}$ & $\begin{array}{l}1 \text { de } 3= \\
33,3 \%\end{array}$ & 2 de $3=$ \\
\hline 6 & Arqueología & 1 & 1997 & & 0 & 0 & 0 \\
\hline 7 & Dibujo & 1 & 2011 & & $\begin{array}{c}1 \text { de } 1= \\
100 \%\end{array}$ & $\begin{array}{c}1 \text { de } 1= \\
100 \%\end{array}$ & 0 \\
\hline 8 & $\begin{array}{c}\text { Estética y teoría } \\
\text { de las Artes }\end{array}$ & 3 & 2004 & & $\begin{array}{l}1 \text { de } 3= \\
33,3 \%\end{array}$ & $\begin{array}{l}1 \text { de } 3= \\
33,3 \%\end{array}$ & 0 \\
\hline 9 & $\begin{array}{c}\text { Comunicación Audiovisual } \\
\text { y publicación }\end{array}$ & 4 & 1989 & & $\begin{array}{c}3 \text { de } 4= \\
75 \%\end{array}$ & $\begin{array}{c}2 \text { de } 4= \\
50 \%\end{array}$ & $\begin{array}{c}2 \text { de } 4= \\
50 \%\end{array}$ \\
\hline 10 & Antropología social & 1 & 1995 & & $\begin{array}{c}1 \text { de } 1= \\
100 \%\end{array}$ & $\begin{array}{c}1 \text { de } 1= \\
100 \%\end{array}$ & $\begin{array}{c}1 \text { de } 1= \\
100 \%\end{array}$ \\
\hline 11 & $\begin{array}{l}\text { Composición } \\
\text { arquitectónica }\end{array}$ & 1 & 1998 & & 0 & 0 & $\begin{array}{c}1 \text { de } 1= \\
100 \%\end{array}$ \\
\hline
\end{tabular}

Tabla 3. Datos globales de las revistas incluidas en el área temática de Bellas Artes, en RESH (2015) Fuente: elaboración propia.

En la recogida y el análisis de estos datos, nos sucede igual que en DICE, es decir, que hay revistas que no están categorizadas en ninguna de las once áreas temáticas establecidas. Centrándonos en el caso de RESH, el sistema de clasificación difiere del de DICE, realizando una clasificación desde las humanidades. Desde esta perspectiva, el área de las bellas artes está bien posicionada en cuanto a las variables de análisis: área temática, número de revistas, año de comienzo de revista más antigua, porcentaje de cumplimiento de periodicidad, porcentaje de evaluadores externos, porcentaje de revistas incluidas en Latindex, porcentaje de revistas incluida en alguna base de datos internacional. Ahora bien, si las revistas clasificadas como de bellas artes se taxonomizan como lo hemos hecho en la tabla anterior de DICE, la valoración cambia absolutamente, y nos encontramos con un panorama similar al de DICE en nuestra área de interés de las bellas artes.

La siguiente base de datos la referenciamos simplemente para constatar la circunstancia en la que se encuentra la investigación académica tradicional en el campo de las bellas artes. 


\section{IN-RECH (ÍNDICE DE IMPACTO REVISTAS ESPAÑOLAS DE CIENCIAS HUMANAS)}

Actividad solo vigente en el tramo 2004-2008 (este es el tramo de la tabla que está en la web). Catalogadas 48 revistas de las bases de datos (de las cuales, gran parte son del campo de la historia del arte).

Comunicado: cese de actualización del producto.

No obstante, de los datos recogidos en ese periodo, podemos decir que en IN-RECH, para analizar el impacto de las publicaciones que tiene en la comunidad científica a través de las citas bibliográficas en el periodo 2004-2008, observamos que el conjunto de revistas con algún impacto es muy reducido y que los índices son significativamente bajos si los comparamos con otras disciplinas humanísticas. La revista que mayor índice presenta es el 0.094 (Revista de Musicología) en el indicador citas/por artículo. El grado de internacionalización es muy bajo, toda vez que en los años referenciados solo hay tres citas internacionales del conjunto global de todas las revistas.

Actualmente, estas son las consultas que se pueden realizar:

- Índice H de las revistas científicas españolas según Google Scholar Metrics (2007-2011, 2008-2012)

- Índice H de las revistas españolas de Ciencias Sociales y Jurídicas según Google Scholar (2001-2010, 2002-2011)

\section{CIRC (CLASIFICACIÓN INTEGRADA DE REVISTAS CIENTÍFICAS)}

Esta es la base de datos de revistas científicas de ciencias sociales y humanas más actual con la que contamos (2. ${ }^{a}$ edición de 2012), que integra los productos de evaluación existentes considerados positivamente por las diferentes agencias de evaluación nacionales como la CNEAl y la Aneca. Esta base de datos recoge 20756 revistas nacionales e internacionales. No realiza una clasificación por materias, sino que estable cuatro categorías: A (Excelente), A, B y C, en relación con el impacto de estas utilizando los criterios de los siguientes productos: Journal Citation Reports; Science Edition, Journal Citation Reports; Social Science Edition, Arts and Humanities Citation Index; Source Publication List, Science Citation Index; Source Publication List, Social Sciences Citation Index; Source Publication List; Índice de impacto de las revistas españolas de ciencias sociales, Índice de impacto de las Revistas Españolas de Ciencias Sociales; Difusión y Calidad Editorial de las Revistas Españolas de Humanidades y Ciencias Sociales; European Reference Index for the Humanities; Latindex; Scopus; List of Titles; y Scientific Journal Rankings.

Curiosamente, de todos estos productos, si prescindimos de Science Citation Index, el total de revistas exclusivamente de ciencias sociales es de 13 281. Dentro de este conjunto, 4833 (36 \%) están clasificadas como revistas Grupo A, 4378 (33 \%) como revistas Grupo B y 4073 (31\%) como revistas Grupo C. Asimismo, de todo este conjunto, 970 revistas, el $7 \%$ del total fueron clasificados como excelente. De cuyo grupo de excelencia, solo podríamos incluir 4 revistas dentro del campo de las artes, siempre que utilizáramos un enfoque 
interdisciplinar: International Journal Art and Design Education, International Review of African American Art, Journal of Aesthetics and Art Criticism y Psychology of Aesthetics Creativity and the Arts. Como palabra clave de búsqueda, hemos utilizado la palabra art, y solo hemos encontrado estas cuatro revistas (véase figura 1 ).
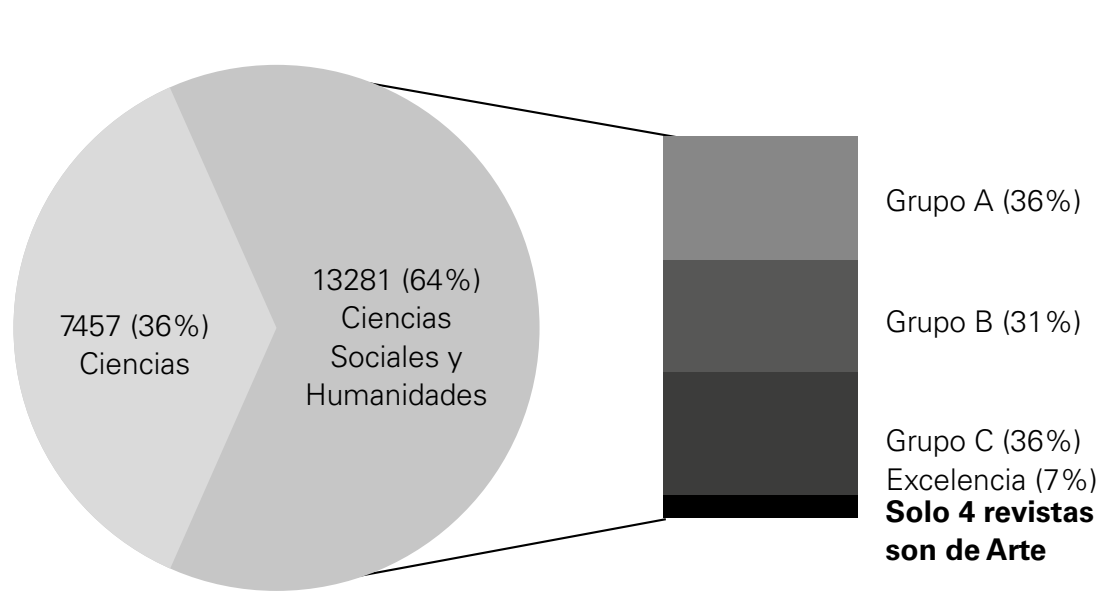

Ciencias

Ciencias sociales Grupo A

Ciencias sociales Grupo B

Ciencias sociales Grupo C

Ciencias sociales Excelencia

Figura 1. Porcentaje de revistas de CIRC por materias.

Fuente: Elaboración propia.

\section{SCOPUS}

En el caso de la base de datos internacionales, hemos recurrido a Scopus. Scimago Journal \& Country Journal nos permite establecer un criterio de discriminación de las revistas que pertenecen al campo de las artes visuales y las artes escénicas (Subject Category: Visual Arts and Performing Arts). La base de datos nos muestra que el impacto de las revistas españolas en el contexto internacional es muy bajo. Solo una revista (Anuario Musical) de las 14 españolas que aparecen en esta categoría está dentro del cuartil 1; cuatro quedan clasificadas en el cuartil 2 (Arte, Individuo y Sociedad, Archivo Español de Arte, Arqueología de la Arquitectura y Lino); el resto de revistas (9) caen a los dos últimos cuartiles. Si analizamos la clasificación que ofrece por países, nos indica que en España hay una considerable producción académica, pues es el sexto país del mundo en la producción de artículos, por encima de países como Alemania e Italia. Es muy significativo que en el campo de las artes tenga una posición tan elevada en el ranking, muy superior si lo comparamos con otros rankings del campo académico. Curiosamente, pese a que el número de revistas del ámbito académico en las bellas artes es bastante recudido en comparación con otras áreas de conocimiento, sí ha subido en los últimos años el número de publicaciones en el campo de las bellas artes. Esto es debido a los procesos de agencialización a los que se ven sometidos los profesores de dicho campo (véase tabla 4). 


\begin{tabular}{|c|c|c|c|c|c|c|c|}
\hline Rank & Country & Documents & $\begin{array}{c}\text { Citable } \\
\text { documents }\end{array}$ & Citaions & $\begin{array}{c}\text { Self }- \\
\text { Citations }\end{array}$ & $\begin{array}{c}\text { Citations } \\
\text { per } \\
\text { Document }\end{array}$ & $\begin{array}{c}\text { H } \\
\text { index }\end{array}$ \\
\hline 1 & United States & 14181 & 12952 & 21657 & 3338 & 3,82 & 45 \\
\hline 2 & $\begin{array}{c}\text { United } \\
\text { Kingdom }\end{array}$ & 6420 & 5859 & 6002 & 1597 & 1,6 & 20 \\
\hline 3 & France & 1795 & 1710 & 926 & 148 & 1,55 & 11 \\
\hline 4 & Canada & 1752 & 1621 & 2276 & 295 & 2,72 & 18 \\
\hline 5 & Australia & 1597 & 1511 & 1769 & 428 & 1,87 & 15 \\
\hline $\mathbf{6}$ & Spain & $\mathbf{1 3 9 3}$ & $\mathbf{1 3 3 8}$ & $\mathbf{4 5 7}$ & $\mathbf{1 2 0}$ & $\mathbf{0 , 7 3}$ & $\mathbf{9}$ \\
\hline 7 & Germany & 1294 & 1232 & 1269 & 132 & 1,26 & 13 \\
\hline 8 & Italy & 1110 & 1066 & 632 & 161 & 0,84 & 9 \\
\hline 9 & Netherlands & 699 & 641 & 684 & 92 & 1,44 & 10 \\
\hline 10 & South Korea & 575 & 513 & 167 & 9 & 1,09 & 6 \\
\hline
\end{tabular}

Tabla 4. Clasificación por países de Scopus. Categoria: Visual Arts and Performing Arts. Periodo: 1996-2013. Fuente: Scopus. Scimago Journal \& Country Journal.

La debilidad de la evaluación de la calidad de la investigación de las revistas españolas no proviene, por tanto, de la cantidad, sino de la calidad de las publicaciones y su débil impacto. Tal como observamos en la tabla el Índice $\mathrm{H}$ es similar al de los países no anglosajones, pero el porcentaje de citas por artículo es el menor de los diez primeros países. Ahora bien, el indicador que nos ofrece una ineficiencia de las publicaciones es el que corresponde a la columna de citas por documento. De hecho, si establecemos una clasificación de las citaciones por documento, España aparecería en el puesto 61, con un valor de 0.73 por detrás de todos los países europeos y de otros países no desarrollados (véase figura 2).

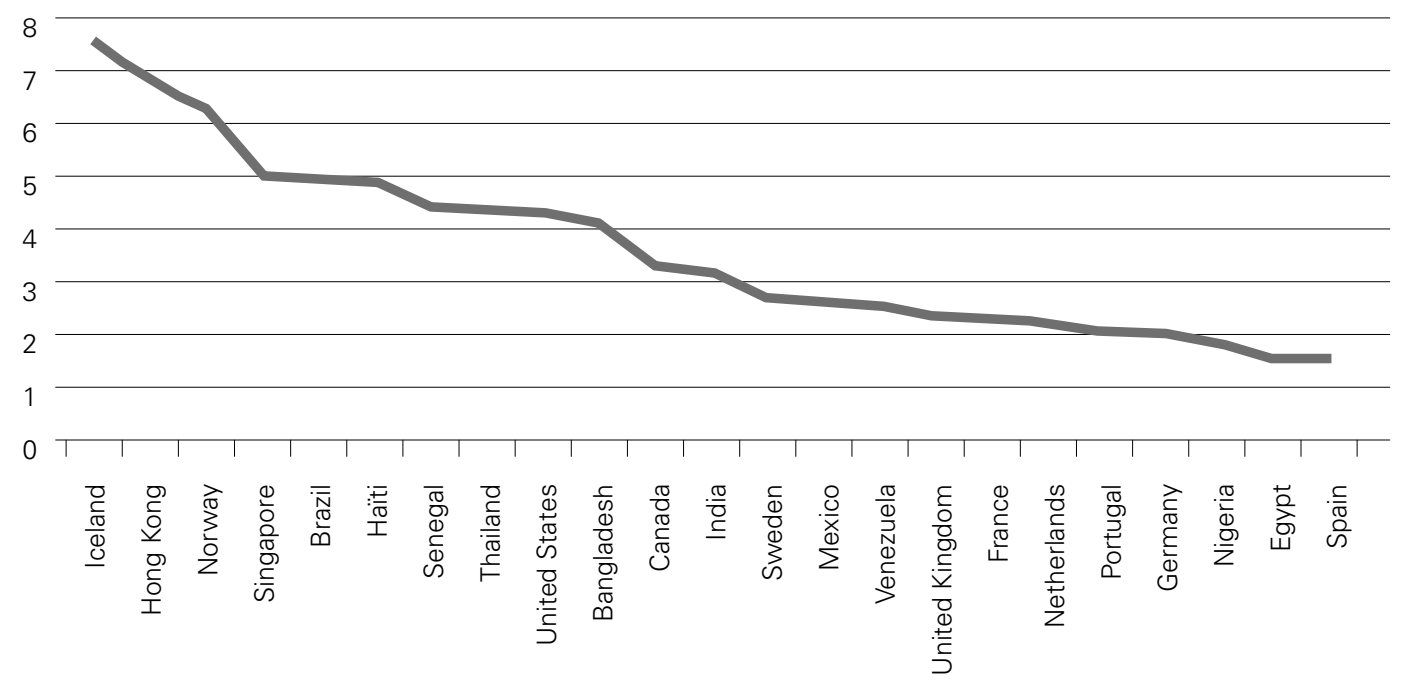

Figura 2. Ratio citas/documentos de algunos países de acuerdo con la clasificación establecida por Scopus. Fuente: Scopus y Scimago Journal \& Country Journal. 


\section{LA INVESTIGACIÓN BASADA EN EL PROYECTO Y LA INVESTIGACIÓN BASADA EN LA PRÁCTICA ARTÍSTICA: UNA EVALUACIÓN DESDE LAS REGLAS DEL CAMPO ARTÍSTICO}

En el primer epígrafe de este trabajo, identificábamos una corriente de pensamiento en el siglo XX, iniciada por Dewey, en la que establecía una analogía entre el ethos de los artistas y los científicos en cuanto a sus actitudes y sus métodos. En ambos casos, tratan de conformar una mirada del mundo con arreglo a sus recursos cognitivos, procedimentales y actitudinales a través de una obra o de una publicación. Por tanto, en este epígrafe, nos alejamos de las disciplinas académicas que "piensan" el arte, como la historia, la sociología, la filosofía, para centramos en la investigación sobre el proceso de trabajo del artista, sobre la obra. Las agencias como la Aneca o la CNEAl, que determinan en buena medida el capital académico-investigador de los docentes, reconocen las obras artísticas como méritos investigadores si bien hasta el momento de una forma muy primaria y sin el desarrollo y recorrido que ha tenido la bibliometría y la medición del impacto académico. Esta es la cuestión clave de los "Indicadores para la evaluación de la actividad investigadora en los ámbitos de las bellas artes" (2011): se parte del diagnóstico de que "el ámbito de la creación y producción artística no cuenta, a nuestro entender, con criterios de valoración adecuados" (16) y se reconoce que existe en el campo de las artes un notable contraste entre las prácticas de los docentes y su evaluación como méritos de investigación. Como consecuencia de ello, se constata un significativo porcentaje de profesores que desarrollan sus procesos creativos (dibujo, pintura y escultura) que no se someten a evaluación de la actividad investigadora por parte de las agencias. Asimismo, se identifican otros factores que contribuyen a una minusvaloración del campo de las artes por parte de las agencias en lo que se refiere al escaso peso de las humanidades en la financiación pública, la dificultad para logar la financiación privada y el menor peso que tienen las humanidades en el CSIC.

Los "Indicadores para la evaluación de la actividad investigadora en los ámbitos de las bellas artes" (2011) recogen un conjunto de indicadores para valorar los proyectos y las obras artísticas de acuerdo con las siguientes dimensiones:

- Indicios de calidad del proyecto: indicadores que valoran tanto proyectos como obras artísticas, audiovisuales, del diseño y de la conservación de bienes culturales, valorando su relevancia, a través de la cadena de valor del proyecto u obra. Lo importante es que la evaluación se produce dentro de las lógicas de cada campo artístico y la valoración que hacen parte de los distintos subsistemas que se concitan en los mundos del arte y los reconocimientos en forma de financiación (subvenciones públicas, patrocinios) o calidad (premios).

- Indicios del impacto del proyecto: a través de medios de comunicación, redes formales o informales y su difusión.

Esta propuesta de valoración de los méritos artísticos como méritos de investigación no ha tenido el necesario posterior desarrollo entre el colectivo de las facultades desde su formulación en 2011 hasta nuestros días. Sin tener conocimiento de las causas del freno a este proyecto, el futuro desarrollo podría consistir en establecer una analogía entre los proyectos artísticos y las publicaciones en las revistas científicas, desde un enfoque pragmático. 
Así, del mismo modo que el prestigio de las revistas científicas es un indicador directo del artículo publicado, en el caso de los méritos artísticos podrían ser evaluados en función de su relación con la institución cultural vinculada al proyecto (por ejemplo una exposición individual sería evaluada en función del prestigio del espacio expositivo de la institución cultural). Para ello, se podría establecer un sistema de objetivación de calidad de las instituciones artísticas españolas e internacionales. Asimismo, se propondrían otras formas complementarias de valorar el impacto artístico en relación con la percepción de los stakeholders de los campos artísticos y del propio mercado del arte. Ello contribuiría a mejorar la evaluación de la actividad investigadora de los profesores docentes por parte de las agencias del sistema universitario español. En este sentido, la teoría de los campos de Bourdieu (1995) nos permite establecer y evidenciar aquellos artistas e instituciones que tienen una valoración y una posición dominante en cada disciplina artística y ello tendría una consecuencia directa en la evaluación de la investigación para estas dos dimensiones de la investigación: la investigación basada en el proyecto y la investigación basada en la práctica artística.

\section{CONCLUSIONES}

Para poder evaluar la calidad de la investigación en el campo de las artes, hay que reconocer un a priori conceptual que se refiere a su naturaleza epistémica, práctica y experiencial. Pasar por alto este a priori tiene como resultado que las evaluaciones institucionales no tengan la congruencia ni el propio reconocimiento de los propios sujetos evaluados, en este caso, los profesores adscritos a las facultades y departamentos de arte de las universidades. El devenir de los enfoques metodológicos de las ciencias sociales en el siglo XX ha conllevado nuevas formas de acercamiento a la investigación en las artes y la adscripción de las facultades de artes a la universidad en algunos países europeos como España cambian por completo el escenario y el statu quo de los profesores artistas, pues necesitan desde su adscripción adecuarse a las normas, los valores y las prácticas del resto de disciplinas académicas. Ello ha conllevado una transición que aún perdura en nuestros días, toda vez que la agencialización del campo académico se ha producido a inicios del siglo XXI en España. En este trabajo, se contextualizó y evidenció esta transición tomando como eje de referencia la evaluación de la investigación y sus indicadores calidad y la teoría de los campos del sociólogo francés Pierre Bourdieu por varias razones: 1) por la pertinencia de su teoría a la hora de analizar desde una perspectiva sociológica tanto el campo académico como los diferentes campos artísticos y 2) por la enorme influencia que su teoría ha tenido en la literatura científica de finales del siglo XX y en la actualidad en el campo de la sociología del arte, la sociología de la educación y en los procesos de institucionalización de la cultura y de la ciencia en la sociedad contemporánea. Asimismo, hemos recurrido a otras teorías de menor alcance, tales como la teoría de la agencialización, para evidenciar, en el caso español, cómo se ha implementado en el campo académico y, asimismo, para observar ciertos resultados que se refieren a las implicaciones en el comportamiento de las propias universidades, además de los profesores. También hemos analizado los resultados de los indicadores bibliométricos en diferentes bases de datos nacionales e internacionales, con el fin de mostrar que objetivamente no son muchas las revistas en español que tengan calidad susceptible para las publicaciones de la investigación 
en las artes, si bien la debilidad de la evaluación de la calidad de la investigación de las revistas españolas no proviene de la cantidad, sino de la calidad de las publicaciones y su débil impacto. Tanto los valores que se aportan del Índice H como del indicador citas/ documento demuestran esta tesis.

Con el objetivo de encajar el ethos de los profesores artistas con una evaluación coherente de su investigación, hemos esbozado la necesidad de plantear un método de evaluación que ha surgido por parte del propio sistema académico a través de los decanos de las facultades de bellas artes españolas — pero que no ha sido desarrollado- que recoja las dimensiones de la investigación basada en el proyecto y la investigación basada en la práctica artística que no pueden acometerse desde el enfoque mainstream de las ciencias. Por ello, acudimos a la propia valoración desde las dinámicas de valoración que se producen dentro del campo artístico desde el enfoque de los campos de producción artística de Bourdieu como un recurso teórico para poder desplegar un conjunto de indicadores consistente, que provenga de los propios campos artísticos y de otros ámbitos conexos como los que se refieren a los indicadores de impacto en otros subsistemas sociales.

\section{NOTAS}

1 Real Decreto 968/1978, sobre transformación de las Escuelas Superiores de Bellas Artes de Barcelona, Bilbao, Madrid, Sevilla y Valencia en Facultades de las respectivas Universidades.

\section{REFERENCIAS}

AA. W. "Indicadores para la evaluación de la actividad investigadora en los ámbitos de las bellas artes. Proyecto presentado por la Conferencia de Decanos de las facultades de bellas artes del Estado español" (manuscrito inédito, 2011).

Alcaín Partearroyo, María Dolores, Adelaida Román Román y Elea Giménez Toledo. 2008. “Categorización de las revistas españolas de ciencias sociales y humanas en RESH". Revista Española de Documentación Científica 31 (1): 85-95.

Barrenechea Feldman, Julieta, Javier Castro Spila y Andoni Ibarra Unzueta. 2009. Calidad relacional y evaluación integral de la actividad científica en ciencias sociales y humanidades: propuesta de metodológica e indicadores. Bilbao: Universidad del País Vasco.

Borgdorff, A. 2010. "El debate sobre la investigación en las artes". Cairon: revista de ciencias de la danza 13: 25-46. 
Bourdieu, Pierre. 1995. Las reglas del arte: génesis y estructura del campo literario. Anagrama.

Bourdieu, Pierre. 2008. Homo academicus. Barcelona: Siglo XXI.

Caduff, Corina, Fiona Siegenthaler y Tan Wälchli, eds. 2010. Art and Artistic Researhc: Music, Visual Arte, Design, Literature, Dance. Zürich: Verlag Scheidegger \& Spiess.

Collins, Randall. 2000. The Sociology of Philosophies: A Global Theory of Intelectual Change. Cambridge: Harvard University Press.

Dewey, John. 2008. El arte como experiencia. Barcelona: Paidós.

Eisner, Elliot. 2008 "Art and Knowledge". En Handbook of the Arts in Qualitative Research: Perspectives, Methodologies, Examples, and Issues, editado por J. Gary Knowles y Andra L. Cole, 3-12. Londres: SAGE.

Hernández Hernández, Fernando. 2008. "La investigación basada en las artes: propuestas para repensar la investigación en educación". Educatio Siglo XXI 26: 85-118.

Miller, Arthur. 2007. Einstein y Picasso: el espacio, el tiempo y los estragos de la belleza. Barcelona: Taurus.

Ortega y Gasset, José. 2006. Obras completas. T. VI. Barcelona: Taurus.

Pastor Ruiz, Fátima, Susana Torrado Morales, José Ignacio Gil Felipe y María Nieves Lorenzo Escolar. 2013. "Análisis de las publicaciones del personal docente e investigador de las facultades españolas de Bellas Artes". Revista Española de Documentación Científica 36 (3): 1-8.

Sacristán Adinolfi, Vera. 2014. “Evaluación de la investigación: el declive de un modelo exitoso". RES: Revista Española de Sociología 21: 149-158.

Sanz Menéndez, Luis. 2014. "La evaluación de la ciencia y la investigación". RES: Revista Española de Sociología 21: 137-148.

Serra, Albert. 2007. "Gestió per resultats en les administracions públiques". En Els escenaris de la gestió pública del segle XXI, editado por Francisco Longo y Tamyko Ysa Figueras, 99-132. Barcelona: Escola d’Administració Pública.

Sullivan, Graeme. 2004. Art Practice as Research Inquiry in the Visual Arts. Nueva York: Teachers College, Columbia University.

Talbot, Colin et al. 2000. The Idea of Agency: Researching the Agencification of the (Public Service) World. Washington DC: American Political Studies Association Conference.

\section{Cómo citar:}

Rubio Arostegui, Juan Arturo y Raquel Caerols Mateo. 2018. “La evaluación holística de la investigación en las artes en España: la necesidad y complejidad de confluir las dinámicas de los campos académico y artístico". Cuadernos de Música, Artes Visuales y Artes Escénicas 13 (2): 237-255.

http://doi.org/10.11144/javeriana.mavae13-2.lehd 